\title{
MiR-3146 Induces Neutrophil Extracellular Traps to Aggravate Acute Gouty Arthritis
}

\section{Lizhen Shan}

The Second Affiliated Hospital,Zhejiang University School of Medicine

\section{Di Yang ( $\nabla$ yangdi4126@126.com )}

Zhejiang Provincial People's Hospital,People's Hospital of Hangzhou Medical College https://orcid.org/0000-0003-1124-2219

\section{Fabo Feng}

Zhejiang Provincial People's Hospital

\section{Danjie Zhu}

Zhejiang Provincial People's Hospital

\section{Xiaolin Li}

Zhejiang Provincial People's Hospital

\section{Research Article}

Keywords: acute gouty arthritis, miR-3146, neutrophil extracellular traps, oxidative stress, SIRT1

Posted Date: March 12th, 2021

DOl: https://doi.org/10.21203/rs.3.rs-280306/v1

License: (c) (i) This work is licensed under a Creative Commons Attribution 4.0 International License.

Read Full License 


\section{Abstract}

MiR-3146 plays an important role in the formation of neutrophil extracellular traps (NETs) during the pathogenesis of acute gouty arthritis (AGA). The aim of our study was to explore the underlying role and molecular mechanism of miR-3146 in the formation of neutrophil extracellular traps (NETs) during the pathogenesis of acute gouty arthritis (AGA). The expression of miR-3146 and sirtuin 1 (SIRT1) was determined by real-time PCR and western blot. The luciferase reporter assay was performed to identify the targeting relationship between miR-3146 and SIRT1. Reactive oxygen species (ROS) production was detected by fluorescent staining. NETs formation was demonstrated via immunofluorescence staining and ELISA method. AGA model was induced in rats to verify the effects of miR-3146 inhibition on histopathological changes and NETs. Here, we found miR-3146 expression was dramatically increased in neutrophils of patients with AGA, presenting higher levels of NETs. Monosodium urate (MSU) crystals significantly increased miR-3146 expression and ROS production in neutrophils. The NETs process was also triggered by MSU crystals. Furthermore, we verified the interaction between miR-3146 and SIRT1. Additionally, antagomir-3146-based therapy effectively inhibited the formation of NETs in rats with AGA. MiR-3146-mediated NETs formation may play a potential role in the pathogenesis of AGA.

\section{Introduction}

Gout is a heterogeneous disease characterized by chronic hyperuricemia resulting from the deposition of monosodium urate (MSU) crystals in joints, kidneys, and other soft tissues [1]. Acute gouty arthritis (AGA) is a common rheumatic disease featured with inflammatory cell infiltration, joint swelling and severe pain [2]. However, due to the self-limited nature and short course of AGA, patients often fail to receive conventional urate-lowering therapy after pain relief, resulting in repeated attacks of arthritis. Therefore, the effective therapeutic strategies of AGA based on the underlying mechanism of bone destruction caused by MSU crystals are current research hotspot.

With the deepening of research, the discovery of neutrophil extracellular traps (NETs) and oxidative stress opens a new perspective to understand the role of neutrophils in the pathogenesis of AGA. NETosis is a form of neutrophil death that is distinguished from apoptosis or necrosis in response to pathogens or other irritants [3]. NETs are extracellular structures with various biological activities, which are released by apoptotic neutrophils [4]. In the course of AGA, the formation of early NETs increases the inflammatory response. The rapid chemotaxis of neutrophils to inflammatory sites to aggregate NETs (aggNETs), leading to the degradation of inflammatory cytokines through a variety of hydrolases, indicating that NETosis is involved in the pathogenesis of AGA [5]. Oxidative stress is an early event of AGA that can damage different biomolecules, inhibit hyperblastosis, and cause cell membrane destruction and cell death and refers to elevated reactive oxygen species (ROS) levels [6]. It has been previously shown that NET formation is enhanced in the presence of ROS $[7,8]$.

MiRNAs are noncoding small RNAs containing endogenous 21-23 nucleotides, which are involved in the regulation of eukaryotic gene expression via post-transcriptional levels by binding to $3^{\prime}$-UTR of mRNA of 
their target genes [9]. Recently, a number of miRNAs have been implicated in inflammatory arthritis, including AGA and have been identified as significant biomarkers for the diagnosis and treatment of AGA $[10,11]$. Liu et al reported that the expression of miR-3146 was upregulated in the plasma of AGA patients compared with asymptomatic hyperuricemia group [12]. However, there is no evidence to support that miR-3146 is involved in MSU crystals-induced NETosis. Herein, we mainly explored the functional role of miR-3146 in the pathogenesis of AGA.

\section{Materials And Methods}

\section{Patients}

We recruited a total of 30 male patients with AGA (30-65 years old) according to the 2011 recommendations for the diagnosis and management of gout and hyperuricemia [13], from January 2018 to December 2018 in our hospital. The 30 gouty patients consisted of 17 patients with AGA, which was characterized by the presence of arthritis, and 13 patients with intercritical gout, which was characterized by a confirmed history of gout, but a current absence of symptoms. Exclusion criteria were: failure to consent to the study; suffering from another clinical condition that causes hyperuricemia or cytokine production, such as hemolytic anemia, myeloproliferative disorders, psoriasis, sarcoidosis, acute or chronic renal failure, alcohol intoxication, diabetic ketoacidosis, lactic acidosis, glycogen storage disease type I, hypo- or hyperparathyroidism, or concurrent infections. In addition, 30 age- and gender-

matched healthy subjects (27-60 years old) were enrolled as controls. The healthy controls were selected randomly and had no clinical history of gout, arthritis, or rheumatic disease. Blood samples were collected from each participant.

All experimental protocols were approved by the Ethics Committee of Zhejiang Provincial People's Hospital (Approval number: 2020QT119), and informed consent was obtained from all patients and control subjects.

Whole blood was collected from each subject using heparin anticoagulant tube, centrifuged at $1500 \times \mathrm{g}$ for 10 min at $4^{\circ} \mathrm{C}$ and the plasma thus obtained was frozen at $-80^{\circ} \mathrm{C}$ for the following experiments.

\section{Cell isolation and culture}

Neutrophil fraction was separated from fresh peripheral blood using density gradient centrifugation as previously described [14]. Neutrophils were isolated from heparinized blood using commercially available kit (EasySep Human Neutrophil Enrichment Kit, STEMCELL Technologies Inc, Vancouver, Canada). Briefly, to separate polymorphonuclear leukocytes (PMN) from mononuclear cells (PBMC), a standard procedure of Histopaque-1077 (Sigma-Aldrich Chemical Co, St Louis, USA) gradient density centrifugation was used. After centrifugation the layer of plasma and mononuclear cells was discarded and the remaining erythrocytes/granulocytes were transferred into a fresh tube for erythrocytes lysis with a hypotonic ammonium chloride solution. 
Obtained PMNs were used for the isolation of neutrophils according to neutrophil enrichment kit protocol provided by the manufacturer.

The isolated neutrophils were maintained in RPMI-1640 medium (Gibco, Carlsbad, CA, USA) supplemented with $10 \%$ heat-inactivated fetal bovine serum, $100 \mathrm{U} / \mathrm{ml}$ penicillin and $100 \mu \mathrm{g} / \mathrm{ml}$ streptomycin. Neutrophils $\left(1 \times 10^{7}\right.$ cells $\left./ \mathrm{ml}\right)$ were transfected with miR-3146 mimic, inhibitor, or negative controls or stimulated with $1 \mathrm{mg} / \mathrm{ml}$ monosodium urate (MSU; Sigma-Aldrich, St. Louis, MO, USA) crystals for $2 \mathrm{~h}$ after treatment with or without $10 \mathrm{mM}$ ROS scavenger N-acetyl-L cystein (NAC; Sigma-Aldrich) for $1 \mathrm{~h}$.

\section{In vivo assay}

Adult male Sprague-Dawley rats (160-180g), obtained from Center for Animal Experiment of Zhejiang University School of Medicine were used in this study. The rat model of AGA was established via administration of $200 \mu \mathrm{L} \mathrm{MSU}(20 \mathrm{mg} / \mathrm{mL})$ into the ankle joint cavity. Antagomir-3146 $(10 \mu \mathrm{L})$ or an equal amount of normal saline was injected at the day of modeling.

The animal procedures were approved by the Institutional Animal Care and Use Committee of Zhejiang University School of Medicine (Approval number: 2019-032).

The synovial tissue specimens were removed from the left knee.

\section{Histopathological examination}

The synovial tissues were fixed in 10\% paraformaldehyde overnight before decalcification using ethanol and processed for paraffin embedding. Tissue sections $(5 \mu \mathrm{m})$ were stained with hematoxylin and eosin. Before staining, slides were deparaffinized. To investigate morphologic changes, sections were stained with hematoxylin (Merck, Darmstadt, Germany) and 1\% eosin (Sigma-Aldrich), air-dried, and coverslipped. All slides ( $\times 100$ magnification) were observed and photographed using a microscope equipped with camera (BX51; Olympus Ltd., Tokyo, Japan), and images were analyzed using DP2-BSW software (Olympus Ltd).

\section{Measurement of oxidative indicators}

The indicators of oxidative damage (MDA, SOD and GSH-Px) were measured using the commercial kits (Beijing Boaosen Biotechnology, Ltd., Beijing, China) following the manufacturer's protocols.

\section{Luciferase reporter assay}

HEK-293 cells purchased from the American Type Culture Collection (ATCC, Rockville, MD, USA) were cultured in DMEM supplemented with $10 \% \mathrm{FBS}$ and $100 \mu \mathrm{g} / \mathrm{mL}$ penicillin/streptomycin (all from Gibco, Carlsbad, CA, USA) at $37^{\circ} \mathrm{C}$ with $5 \% \mathrm{CO}_{2}$.

According to the manufacturer's instructions, luciferase assays were performed in HEK-293 cells after cotransfection with the wild/mutated types of SIRT1 promoter reporters and miR-3146 mimic/mimic NC using Lipofectamine 2000. After $48 \mathrm{~h}$, the cells were collected and tested for luciferase activities using the dual-luciferase reporter assay system (Promega, Madison, WI, USA). 


\section{RNA extraction and quantitative real-time PCR}

Total RNA was extracted from cells using TRIzol (Invitrogen, Carlsbad, CA, USA) and converted into cDNA using the PrimeScript RT reagent kit (Takara, Dalian, China) according to the manufacturer's instructions. The relative expression levels of miR-3146 and sirtuin 1 (SIRT1) were measured by real time PCR using SYBR Green I real time PCR kit (BD Biosciences, San Diego, CA, USA) on an ABI 7500 Real-Time PCR system (Applied Biosystems, Carlsbad, CA, USA).

The cycling conditions were as follows: initial denaturation at $95^{\circ} \mathrm{C}$ for $15 \mathrm{sec}$, followed by 45 cycles of $55^{\circ} \mathrm{C}$ for $33 \mathrm{sec}$ and $70^{\circ} \mathrm{C}$ for $30 \mathrm{sec}$.

The relative expressions were calculated through the $2^{-\Delta \Delta C t}$ method. $U 6$ and GAPDH were used as internal controls, respectively. The primers used were as follows: miR-3146, forward: 5'CATGCTAGGATAGAAAGAATGG-3', reverse: universal primers; SIRT1, forward: 5'-

TAGCCTTGTCAGATAAGGAAGGA-3', reverse 5'-ACAGCTTCACAGTCAACTTTGT-3'; U6, forward: 5'AAAGCAAATCATCGGACGACC-3', reverse 5'-GTACAACACATTGTTTCCTCGGA-3'; GAPDH, forward: 5'TGTGGGCATCAATGGATTTGG-3', reverse 5'-ACACCATGTATTCCGGGTCAAT-3'.

\section{Western blot}

Total proteins were extracted from cultured cells using radio-immunoprecipitation buffer (RIPA). Equal amount of denatured protein samples were separated by electrophoresis and transferred to polyvinylidene fluoride membranes. Immunoblotting was performed at $4^{\circ} \mathrm{C}$ overnight with appropriate primary antibodies against SIRT1 (\#9475; Cell Signaling Technology, Boston, MA, USA) at a dilution of 1:1000. After incubating with HRP-conjugated secondary antibodies (\#7074; 1:2000 diltuion, Cell Signaling Technology), the signals were visualized by the ECL system (Thermo Fisher Scientific, Waltham, MA, USA). $\beta$-actin was used as a loading control.

\section{Enzyme-linked immuno sorbent assay (ELISA)}

According to the manufacturer's instructions, the quantification of proinflammatory cytokines was conducted by the commercial ELISA kits (Roche Diagnostics, Indianapolis, IN, USA). The optical absorbance was then measured at $405 \mathrm{~nm}$.

IL-8 and IL-1 $\beta$ concentrations in cell culture supernates and serum were quantified using commercial ELISA kits (human IL-8 and IL-1 $\beta$ ELISA reagent kits from R\&D Systems, Minneapolis, MN, USA; rat IL-8 and IL-1 $\beta$ ELISA reagent kits from R\&D Systems and Shanghai Enzyme-linked Biotechnology Co., Ltd. Shanghai, China), following the manufacturer's instructions. The microplates were sensitized overnight with coating antibody and blocked with a solution of $4 \%$ serine bovine albumin, $5 \%$ sucrose in PBS. Supernatants and serum samples and standards were placed in duplicate in the wells of the plate and incubated at $37^{\circ} \mathrm{C}$ for $1 \mathrm{~h}$. Samples were washed and detection antibody was added and incubated for 1 h. After appropriate washings, streptavidin conjugated to horseradish peroxidase was added and incubation was performed at $37^{\circ} \mathrm{C}$ for $30 \mathrm{~min}$. The reaction was evidenced by the addition of TMB, which 
was incubated for 10 min at $37^{\circ} \mathrm{C}$. The reaction was stopped with $\mathrm{H}_{2} \mathrm{SO}_{4}$ and read at $450 \mathrm{~nm}$ in an ELISA reader (Microplate Reader, SPECTROstarNano, BGM/LABTECH, Ortemberg, Germany).

For myeloperoxidase (MPO)-DNA complex ELISA assay as previously described [15], a "sandwich" ELISA with anti-MPO monoclonal antibody (Merck Millipore Corp., catalog no. 07-496) and peroxidaseconjugated anti-DNA monoclonal antibody (Roche Diagnostics, Indianapolis, IN, USA; Cell Death Detection ELISA no. 1154467500) was used. Briefly, the wells of microtiter strips were coated with a monoclonal antibody specific for MPO to capture MPO-DNA derived from NETs. A peroxidase substrate was added, which reacted with the bound peroxidase to yield a soluble green product detected at $405 \mathrm{~nm}$. Absorbance readings were proportional to the amount of bound horseradish peroxidase-labeled anti-DNA monoclonal antibody. Results were expressed in arbitrary units.

\section{Immunofluorescence staining}

Synovial tissues were digested in Hank's Balanced Salt Solution (Thermo Fisher Scientific, Waltham, MA, USA) containing $0.25 \%$ trypsin (Gibco) at $37^{\circ} \mathrm{C}$. Treated neutrophils or embedded tissues were fixed in $4 \%$ paraformaldehyde for $15 \mathrm{~min}$ and permeabilized in $0.01 \%$ Triton X-100 for $10 \mathrm{~min}$ at room temperature. Samples were then stained with citrullinated histone H3 (CitH3) (1:50 dilution; Abcam, Cambridge, UK) and LYG6 antibodies (1:100 dilution; eBioscience, San Diego, CA, USA). The nucleus was counter-stained with DAPI. Images were captured by confocal microscopy (Zeiss, Jena, Germany) from five randomly fields. The results were expressed as the number of NETs/50 Ly6G-positive cells.

\section{Measurement of intracellular ROS}

Intracellular ROS accumulation was measured using dichlorofluorescin diacetate (DCFH-DA; SigmaAldrich) as a peroxide-sensitive fluorescent probe at the wavelength $488 / 525 \mathrm{~nm}$ as previously described $[16,17]$. Briefly, DCFH-DA was diluted in serum-free DMEM to a final concentration of $10 \mu \mathrm{M}$ and was added to each well for replacing of original medium. After incubation for $30 \mathrm{~min}$ at $37^{\circ} \mathrm{C}$, cells were washed thrice with serum-free DMEM. The fluorescence intensities were recorded with a spectrophotometer (Shimadzu Corporation, Tokyo, Japan) with an excitation of $485 \mathrm{~nm}$ and an emission of $525 \mathrm{~nm}$.

\section{Statistical analysis}

Data from at least three independent experiments were expressed as mean \pm standard deviation (SD), analyzed by SPSS 19.0 statistical software (SPSS, Chicago, IL, USA). One-way ANOVA was used to analyze the differences between groups. $P<0.05$ indicated a statistically significant difference.

\section{Results}

\section{MiR-3146 is significantly upregulated in patients with AGA}

As shown in Fig. 1A, the expression of neutrophil miR-3146 was markedly upregulated in patients with AGA relative to healthy volunteers. NETs formation, which was assessed by circulating MPO-DNA capture 
ELISA method (Fig. 1B) and immunofluorescence microscopy (Fig. 1C), was significantly increased in AGA cases than that in healthy controls, indicating that NETosis might be involved in the pathogenesis of AGA. The excessive ROS production was observed in AGA patients compared to healthy subjects (Fig. 1D). Compared to healthy controls, the mRNA and protein levels of neutrophil SIRT1 were signally decreased in these AGA patients (Fig. 1E-1F). Furthermore, AGA patients exhibited higher serum levels of proinflammatory cytokines IL-8 and IL-1 $\beta$ compared to healthy individuals (Fig. 1G).

\section{Neutrophil miR-3146 and ROS production are increased in response to MSU crystals}

Our data showed that miR-3146 was memorably upregulated in human neutrophils stimulated with MSU crystals (Fig. 2A). The addition of MSU crystals efficiently induced NET formation of human neutrophils (Fig. 2B-2C). As presented in Fig. 2D, a significantly higher amount of ROS was produced by human neutrophils subjected to MSU exposure compared to the controls. Moreover, the stimulation of MSU crystals resulted in a decrease of SIRT1 mRNA and protein levels in human neutrophils (Fig. 2E-2F). MSU crystals-stimulated neutrophils led to elevated secretion of IL-8, and IL-1ß (Fig. 2G).

\section{MiR-3146 directly targets SIRT1 in neutrophils}

MiR-3146 harbored putative binding sites for SIRT1 (Fig. 3A). We further verified the interaction between miR-3146 and SIRT1 using luciferase reporter assay. As expected, miR-3146 mimic inhibited firefly luciferase expression (Fig. 3B). MiR-3146 overexpression led to SIRT1 downregulation at mRNA and protein levels, while miR-3146 inhibition led to an opposite effect (Fig. 3C-3D).

\section{MSU crystals trigger NETosis via oxidative stress by regulating miR-3146}

To further verify whether miR-3146 was implicated in the process of NETosis, miR-3146 expression was overexpressed in human neutrophils (Fig. 4A). Besides, miR-146a overexpression aggravated ROS excessive generation (Fig. 4B), SIRT1 downregulation (Fig. 4C-4D), NETs formation (Fig. 4E), and IL-8 and IL-1 $\beta$ overproduction (Fig. 4F) induced by MSU crystals. However, treatment with ROS scavenger NAC in human neutrophils effectively abrogated these effects of miR-3146 overexpression.

\section{MiR-3146 aggravates AGA progression via inducing NETs formation}

Rats with AGA maintained arthritis status (Fig. 5A) and exhibited serious swelling (Fig. 5B) compared with the control group, whereas treatment with antagomir-3146 significantly decreased the arthritis score and edema. As demonstrated by ELISA test, the levels of IL-8 and IL-1 $\beta$ were significantly higher in AGA group than that in the sham-operated group, while treatment with antagomir-3146 memorably reduced the secretion of proinflammatory cytokine (Fig. 5C). MDA content was observably increased, while the 
levels of SOD and GSH-Px were markedly attenuated in AGA group compared to the control group. Administration with antagomir-3146 significantly led to an opposite effects (Fig. 5D). As shown in Fig. 5E, AGA rats treated with antagomir-3146 showed mild synovial hyperplasia, inflammation and cartilage erosion as evidenced by hematoxylin/eosin staining. Immunofluorescence confocal microscopy confirmed NETs formation in rats with AGA, and the effects were reversed by the injection of antagomir3146 (Fig. 5F).

\section{Discussion}

In this study, we found that MSU crystals induced NET release from neutrophils via increasing miR-3146 expression. Further in vivo assay showed that the inhibition of miR-3146 prevented AGA progression in experimental rats. Our results provided a novel insight into the contribution of NETosis to the pathogenesis of AGA.

Non-coding RNAs are RNA molecules with no protein-coding potential [18]. MiRNAs are a class of small non-coding RNAs that participate in various physiological or pathological processes [19]. MiRNAs have been implicated as important regulators in the pathogenesis of human inflammatory arthritic diseases, including AGA. For instance, Zhou et al indicated that miR-488 and miR-920 served as anti-inflammatory regulators through IL-1 $\beta$ downregulation in MSU-induced macrophages [20]. Zhang et al implied that miR146a knockout developed the progression of AGA via inhibiting the activation of the NALP3 inflammasome [21]. Herein, upregulated expression of miR-3146 in serum was detected in AGA patients. Additionally, the expression of miR-3146 was higher in human polymorphonuclear neutrophils (PMN) after exposure to MSU crystals in vitro. Thus, miR-3146 might be a potential therapeutic target for AGA.

There is emerging evidence that NETosis is implicated with the occurrence of non-infectious inflammatory diseases $[22,23]$. loannis et al demonstrated for the first time that activation of neutrophils is associated with the formation of proinflammatory NETs stimulated with MSU crystals in gout [24]. The generation of ROS is required for NETosis by the activation of the nicotinamide adenine dinucleotide phosphate (NADPH) oxidase [25]. Indeed, it has recently been shown that MSU crystals induce NETosis in a ROS-dependent manner [26, 27]. In the current research, we showed that the formation of NETs and ROS levels were significantly increased in patients with AGA. Additionally, MSU crystals displayed enhanced NETosis and ROS production released by human PMN, suggesting that MSU crystals triggered NETosis that was dependent on the production of ROS.

To further investigate the molecular mechanism by which miR-3146 contributed to neutrophil-dependent oxidative stress, the potential target genes of miR-3146 were investigated. Here, we found that SIRT1 expression was significantly downregulated in AGA patients and human PMN following treatment with MSU crystals. As a member of histone deacetylase family, SIRT1 is involved in the regulation of various physiological functions, including glucose and lipid metabolism, oxidative stress injury, inhibition of inflammatory response, and cell proliferation, senescence, and apoptosis through deacetylation [28]. Using bioinformatics, luciferase reporter assays, and miRNA overexpression and knockdown techniques, 
we identified that miR-3146 could directly target SIRT1 and thereby suppress the expression of SIRT1. Our data further found that excess ROS generation induced by MSU crystals was decreased by miR-3146 overexpression in human PMN. In contrast, NAC, a ROS scavenger antagonized NETosis and oxidative stress injury induced by miR-3146. Rats with miR-3146 deficiency attenuated MSU crystals-induced synovial inflammation and oxidative damage via dysregulation of NETosis.

\section{Conclusion}

In conclusion, we provided the first demonstration that miR-3146 initiated NETosis in a ROS dependent manner via targeting SIRT1 which ultimately triggered the development of AGA. Accordingly, our data suggest that the inhibition of NETosis might be developed as a powerful treatment strategy of AGA.

\section{Declarations}

\section{Ethics approval and consent to participate}

All procedures were approved by the Animal Care and Use Committee of Zhejiang Provincial People's Hospital.

\section{Consent for publication}

Not applicable.

\section{Availability of data and materials}

The datasets used and analyzed during the current study are available from the corresponding author on reasonable request.

\section{Competing interests}

The authors declare that they have no competing interests.

\section{Funding}

This study was supported by Natural Science Foundation of Zhejiang Province (LY18H060009) and Medical Health Science and Technology Project of Zhejiang Provincial Health Commission (2018KY018).

\section{Authors' contributions}

Lizhen Shan: Data curation, Writing-Original draft preparation. Fabo Feng: Resources, Data Curation. Danjie Zhu:Investigation,Supervision. Xiaolin Li: Software, Validation,Visualization. Di Yang:Conceptualization, Methodology, Writing- Reviewing and Editing.

\section{Acknowledgements}


Not applicable.

\section{References}

1. Hainer, B.L., E. Matheson, and R.T. Wilkes, Diagnosis, treatment, and prevention of gout. Am Fam Physician, 2014. 90(12): p. 831-6.

2. Cleophas, M.C., T.O. Crisan, and L.A. Joosten, Factors modulating the inflammatory response in acute gouty arthritis. Curr Opin Rheumatol, 2017. 29(2): p. 163-170.

3. Masuda, S., et al., NETosis markers: Quest for specific, objective, and quantitative markers. Clin Chim Acta, 2016. 459: p. 89-93.

4. Sørensen, O.E. and N. Borregaard, Neutrophil extracellular traps - the dark side of neutrophils. Journal of Clinical Investigation, 2016. 126(5): p. 1612-1620.

5. Desai, J., S. Steiger, and H.J. Anders, Molecular Pathophysiology of Gout. Trends Mol Med, 2017. 23(8): p. 756-768.

6. Zamudio-Cuevas, Y., et al., Molecular basis of oxidative stress in gouty arthropathy. Clin Rheumatol, 2015. 34(10): p. 1667-72.

7. von Kockritz-Blickwede, M., et al., Phagocytosis-independent antimicrobial activity of mast cells by means of extracellular trap formation. Blood, 2008. 111(6): p. 3070-80.

8. Liao, P., et al., Polydatin effectively attenuates disease activity in lupus-prone mouse models by blocking ROS-mediated NET formation. Arthritis Research \& Therapy, 2018. 20.

9. Wang, Z. and Y. Liu, Predicting Functional MicroRNA-mRNA Interactions. Methods Mol Biol, 2017. 1580: p. 117-126.

10. Wang, Y., et al., Could MicroRNAs be Regulators of Gout Pathogenesis? Cell Physiol Biochem, 2015. 36(6): p. 2085-92.

11. Jin, H.M., et al., MicroRNA-155 as a proinflammatory regulator via SHIP-1 down-regulation in acute gouty arthritis. Arthritis Res Ther, 2014. 16(2): p. R88.

12. Liu, P., et al., Expression of microRNAs in the plasma of patients with acute gouty arthritis and the effects of colchicine and etoricoxib on the differential expression of microRNAs. Arch Med Sci, 2019. 15(4): p. 1047-1055.

13. Hamburger, M., et al., 2011 recommendations for the diagnosis and management of gout and hyperuricemia. Phys Sportsmed, 2011. 39(4): p. 98-123.

14. Najmeh, S., et al., Simplified Human Neutrophil Extracellular Traps (NETs) Isolation and Handling. J Vis Exp, 2015(98).

15. Caudrillier, A., et al., Platelets induce neutrophil extracellular traps in transfusion-related acute lung injury. J Clin Invest, 2012. 122(7): p. 2661-71.

16. Zhang, Y.G., et al., Exosomes derived from oxLDL-stimulated macrophages induce neutrophil extracellular traps to drive atherosclerosis. Cell Cycle, 2019. 18(20): p. 2674-2684. 
17. Gao, H., et al., Exosomal MALAT1 derived from ox-LDL-treated endothelial cells induce neutrophil extracellular traps to aggravate atherosclerosis. Biol Chem, 2020. 401(3): p. 367-376.

18. Yang, J.X., R.H. Rastetter, and D. Wilhelm, Non-coding RNAs: An Introduction. Adv Exp Med Biol, 2016. 886: p. 13-32.

19. Lu, T.X. and M.E. Rothenberg, MicroRNA. J Allergy Clin Immunol, 2018. 141(4): p. 1202-1207.

20. Zhou, W., et al., MicroRNA-488 and -920 regulate the production of proinflammatory cytokines in acute gouty arthritis. Arthritis Res Ther, 2017. 19(1): p. 203.

21. Zhang, Q.B., et al., Mice with miR-146a deficiency develop severe gouty arthritis via dysregulation of TRAF 6, IRAK 1 and NALP3 inflammasome. Arthritis Res Ther, 2018. 20(1): p. 45.

22. Yousefi, S., et al., Untangling "NETosis" from NETs. Eur J Immunol, 2019. 49(2): p. 221-227.

23. Berthelot, J.M., et al., NETosis: At the crossroads of rheumatoid arthritis, lupus, and vasculitis. Joint Bone Spine, 2017. 84(3): p. 255-262.

24. Mitroulis, I., et al., Neutrophil extracellular trap formation is associated with IL-1beta and autophagyrelated signaling in gout. PLoS One, 2011. 6(12): p. e29318.

25. Fuchs, T.A., et al., Novel cell death program leads to neutrophil extracellular traps. J Cell Biol, 2007. 176(2): p. 231-41.

26. Schorn, C., et al., Bonding the foe - NETting neutrophils immobilize the pro-inflammatory monosodium urate crystals. Front Immunol, 2012. 3: p. 376.

27. Tatsiy, O., et al., Cytokine Production and NET Formation by Monosodium Urate-Activated Human Neutrophils Involves Early and Late Events, and Requires Upstream TAK1 and Syk. Front Immunol, 2019. 10: p. 2996.

28. Chang, H.C. and L. Guarente, SIRT1 and other sirtuins in metabolism. Trends Endocrinol Metab, 2014. 25(3): p. 138-45.

\section{Figures}



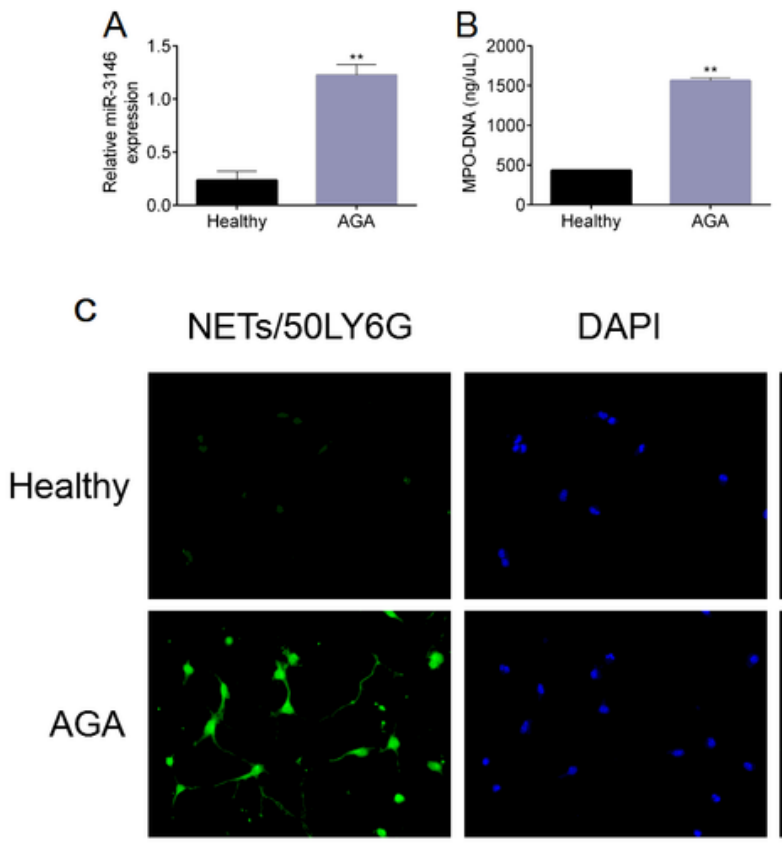

Merge
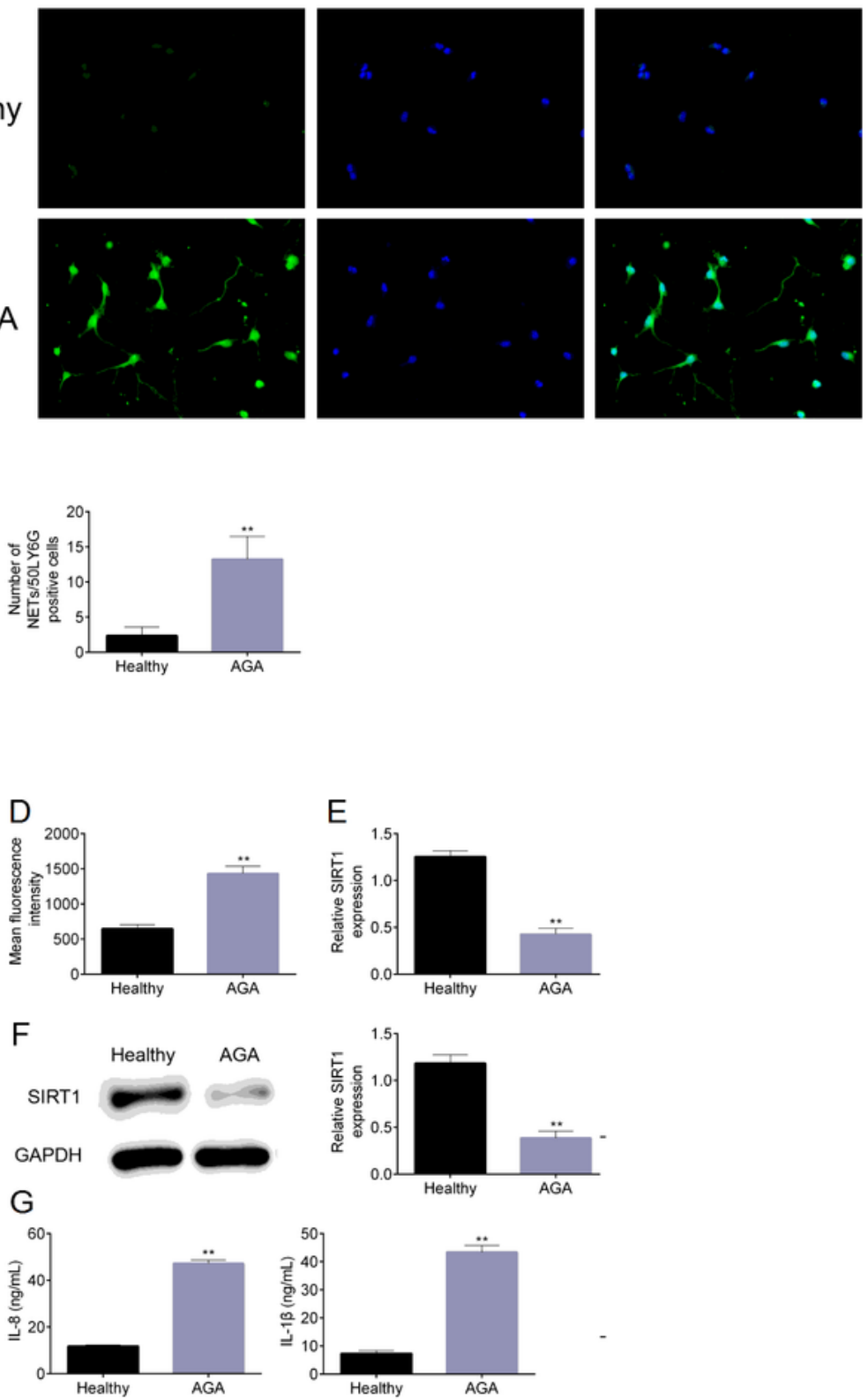

\section{Figure 1}

MiR-3146 is significantly upregulated in patients with AGA The expression of miR-3146 in patients with AGA and healthy volunteers (A); NETs formation was assessed by circulating MPO-DNA capture ELISA method (B) and representative immunofluorescence staining of Ly6G (scale bars show 25-mm intervals; magnification $\times 40)(C)$; The ROS production (D), the mRNA and protein levels of SIRT1 (E-F) and the 
serum levels of IL-8 and IL-1 $\beta(G)$ were determined. Data from at least three independent experiments were expressed as mean $\pm S D$. ${ }^{*}{ }^{*}<<0.01$ vs. healthy group.
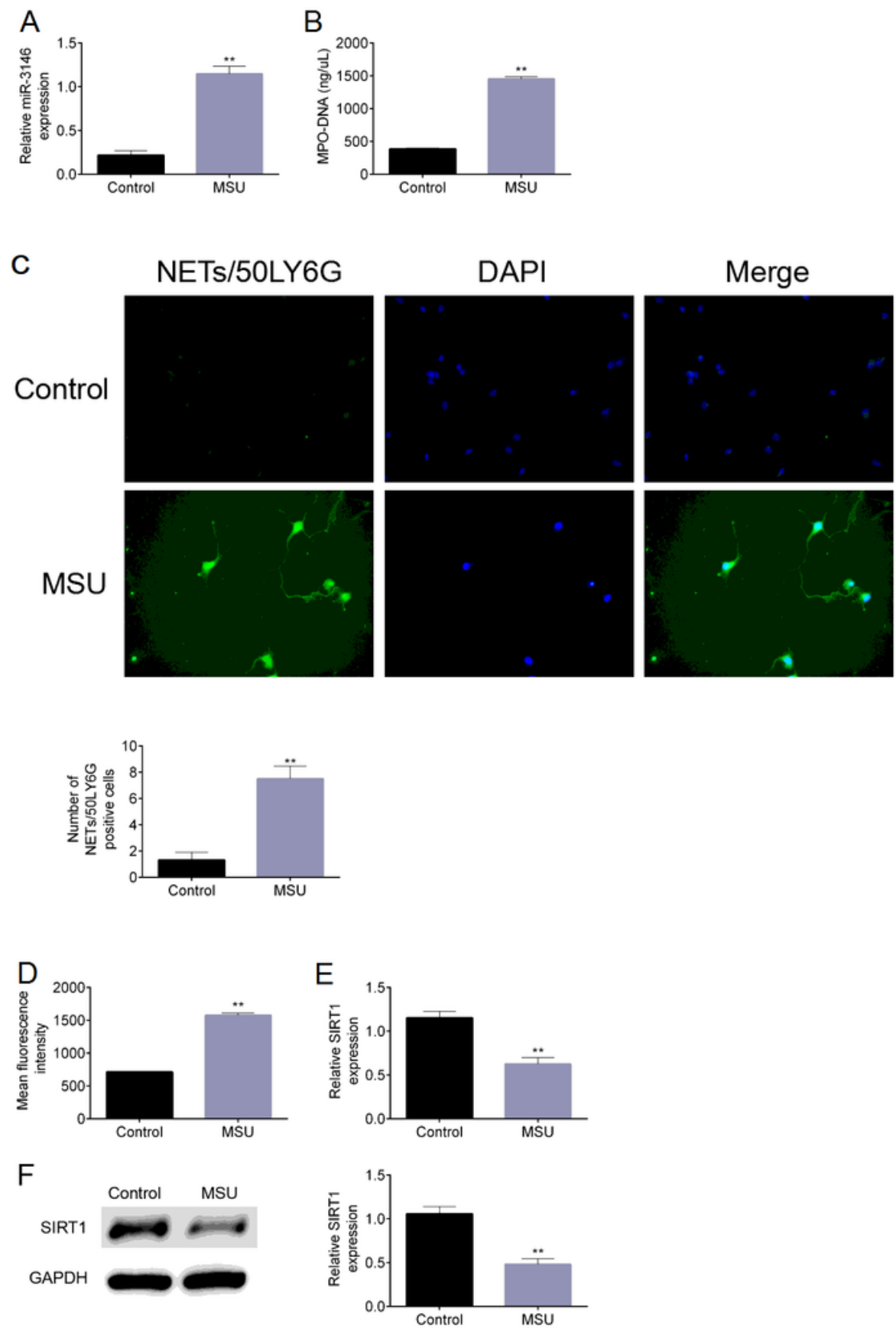

G
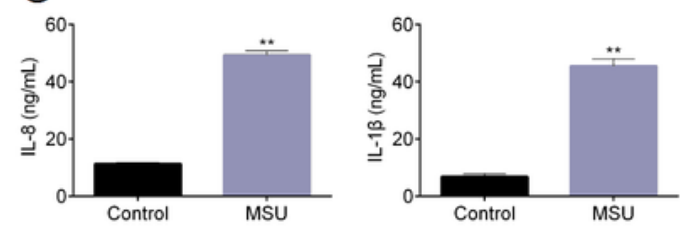

Figure 2

Neutrophil miR-3146 and ROS production are increased in response to MSU crystals The expression of miR-3146 (A), NETs formation (B-C), ROS production (D), the mRNA and protein levels of SIRT1 (E-F) and 
the levels of IL-8 and IL-1 (G) in human neutrophils stimulated with or without MSU crystals. Data from at least three independent experiments $(n=3)$ were expressed as mean $\pm S D$. ${ }^{*} P<0.01$ vs. control group.
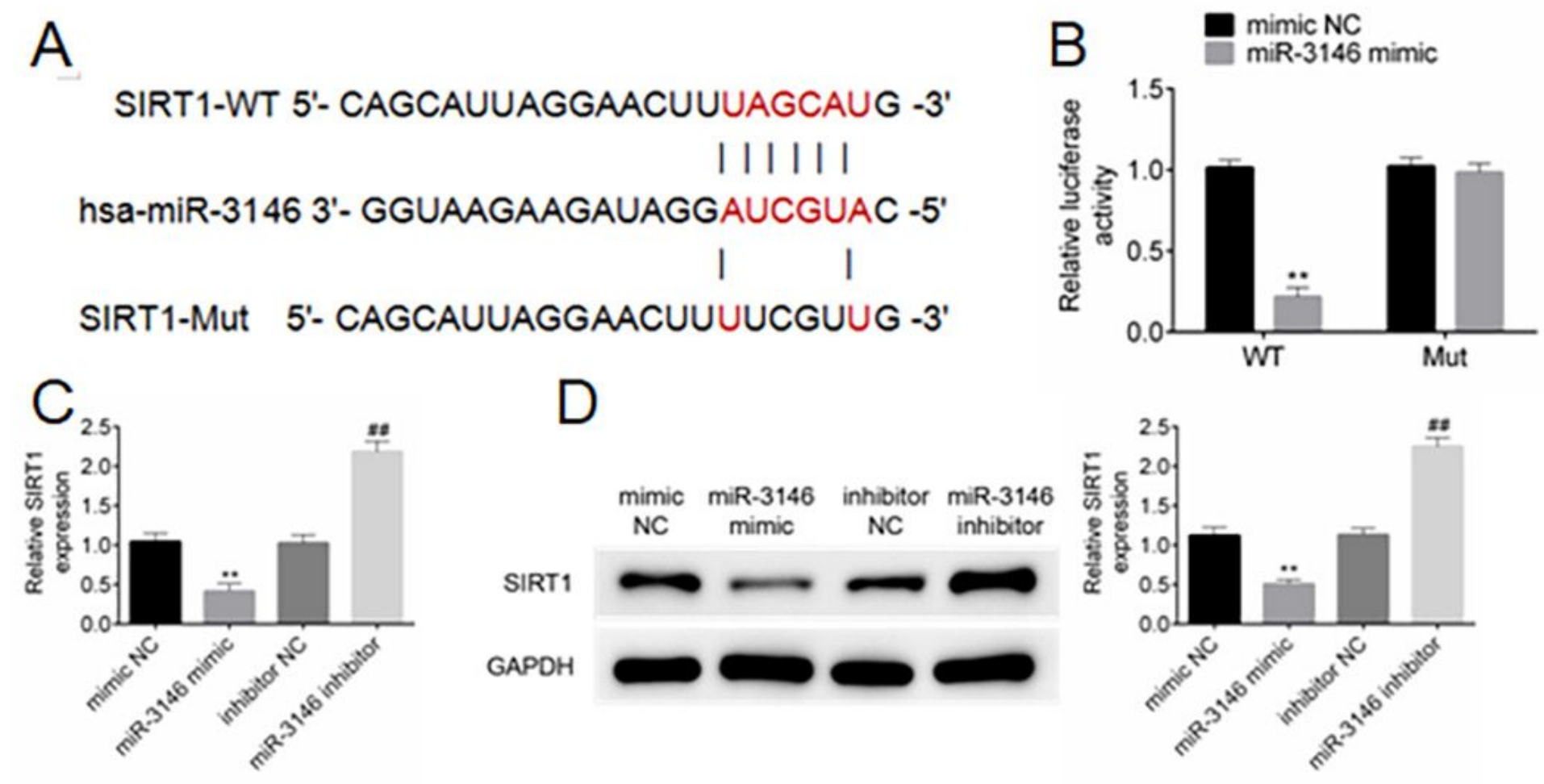

\section{Figure 3}

MiR-3146 directly targets SIRT1 in neutrophils SIRT1 mRNA 3'UTR contained miR-3146 binding sites predicted by TargetScan (A); The luciferase activities were measured in human neutrophils transfected with the wild or mutant type SIRT1 plasmids (B). The mRNA and protein levels of SIRT1 were measured in human neutrophils transfected with miR-3146 mimic or inhibitor (C-D). Data from at least three independent experiments $(n=3)$ were expressed as mean \pm SD. ${ }^{\star *} P<0.01$ vs. mimic NC group; \#\#P<0.01 vs. inhibitor NC group. 


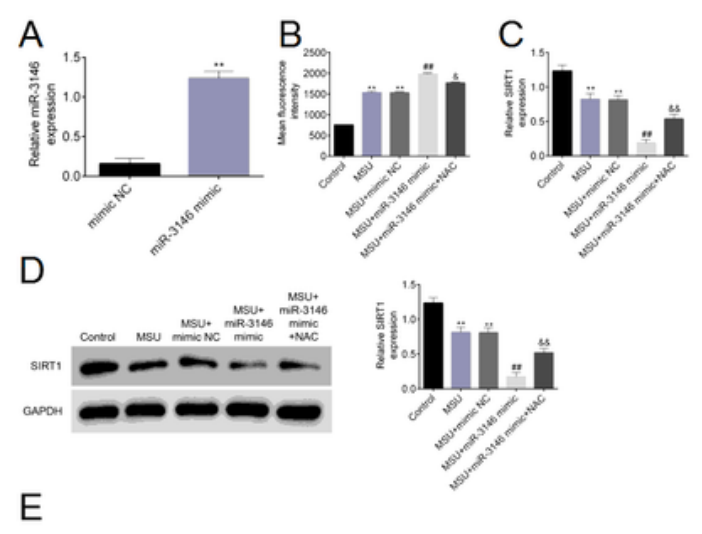

MSU+
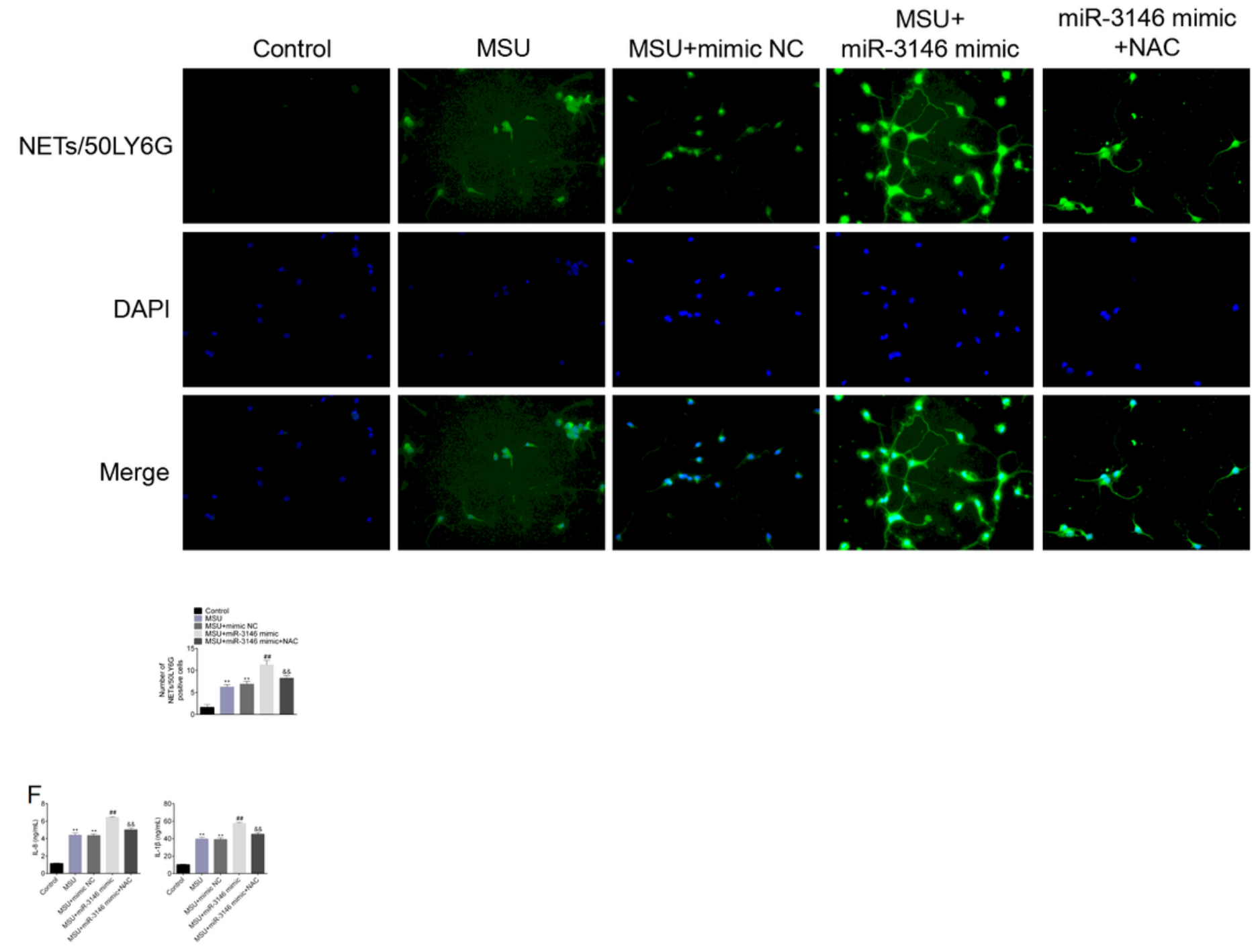

Figure 4

MSU crystals trigger NETosis via oxidative stress by regulating miR-3146 The expression of miR-3146 (A), ROS production (B), the mRNA and protein levels of SIRT1 (C-D), NETs formation (E), and the levels of IL-8 and IL-1 $\beta(F)$ in human neutrophils stimulated with MSU crystals or NAC following transfection with miR3146 mimic. Data from at least three independent experiments $(n=3)$ were expressed as mean \pm SD. 
${ }^{*} \mathrm{P}<0.01$ vs. Control group; \#\#P<0.01 vs. MSU+mimic NC group; \&P<0.05, \&\&P<0.01 vs. MSU+ miR-3146 mimic group.
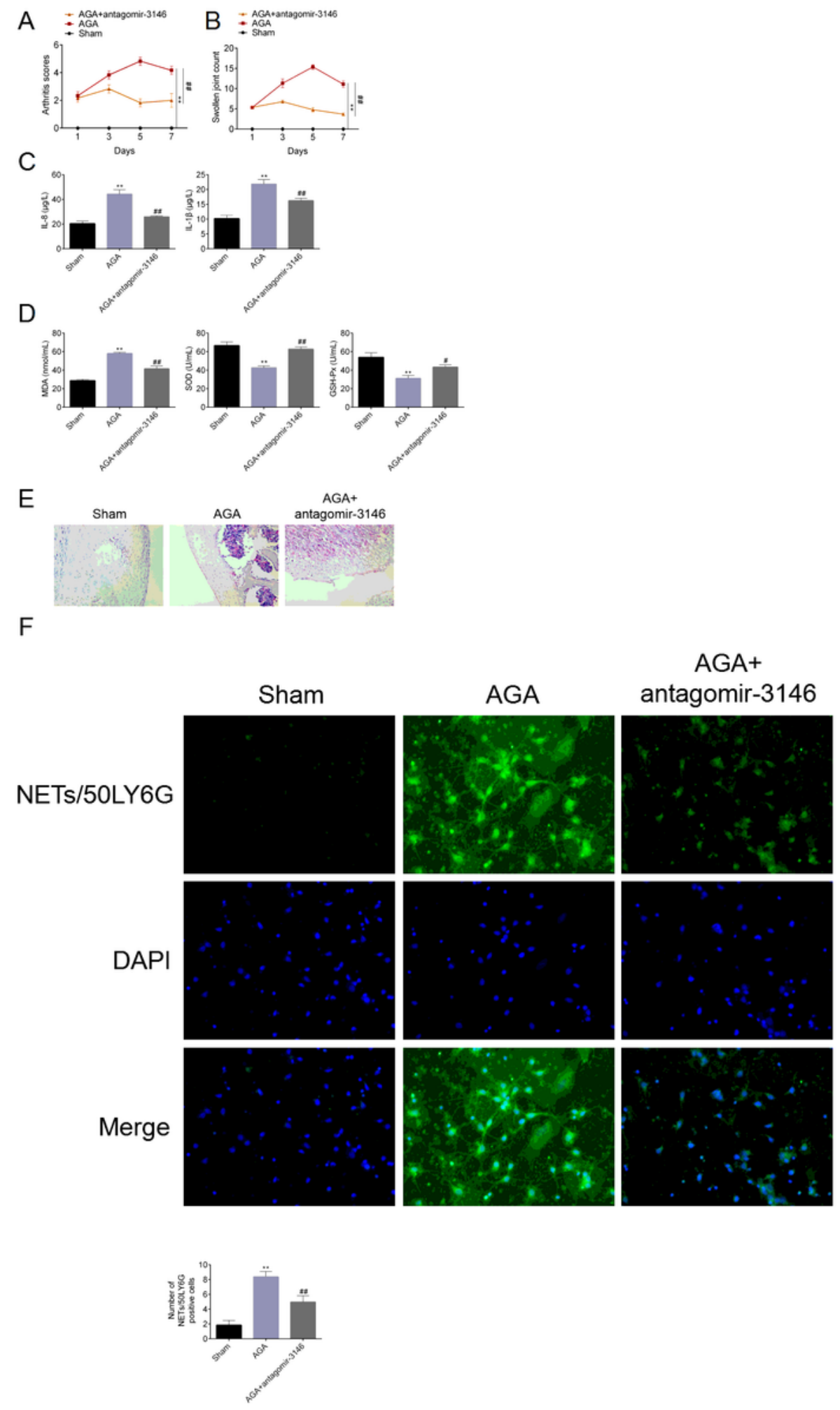

\section{Figure 5}

MiR-3146 aggravates AGA progression via inducing NETs formation Swollen joint count (A), arthritis index (B), the serum levels of IL-8 and IL-1 $\beta(C)$, the levels of MDA, SOD and GSH-Px (D), histopathological examination of synovial tissues (E) and NETs formation (F) in sham-operated rats or 
AGA rats injected with or without antagomir-3146. Data from at least three independent experiments $(n=3)$ were expressed as mean $\pm S D$. ${ }^{*} P<0.01$ vs. Sham group; $\# P<0.01, \# \# P<0.05$ vs. AGA group.

\section{Supplementary Files}

This is a list of supplementary files associated with this preprint. Click to download.

- additionalfile.xIsx 